\title{
A BOUNDED DETERIORATION PROPERTY OF A SYMMETRIC POSITIVE DEFINITE CLASS OF NEWTON-LIKE METHODS AND ITS APPLICATION
}

\author{
Hiroshi Yabe \\ Science University of Tokyo
}

\author{
Naokazu Yamaki \\ Shizuoka University
}

(Received December 16, 1997; Final July 7, 1999)

\begin{abstract}
This paper is concerned with Newton-like methods for solving unconstrained minimization problems. We derive a general form and its factorized form of a symmetric positive definite matrix that satisfies the secant condition in order to approximate the Hessian matrix of the objective function. We obtain the bounded deterioration property for such a general form, which is an extention of the bounded deterioration property for secant methods. Applying the general form to the secant method, we obtain a new family that includes the Broyden family, and we show local and q-superlinear convergence of our method. Furthermore, we propose applying the general form to nonlinear least squares problems to obtain a modification of the Gauss-Newton method.
\end{abstract}

\section{Introduction}

In this paper, we consider the following unconstrained minimization problem:

[UMP]

$$
\text { minimize } f(x), \quad f: R^{n} \rightarrow R, \quad x \in R^{n} .
$$

Let $x_{*}$ be a solution to the problem. There are several numerical methods for solving this problem. The methods of interest are iterative methods that are based on Newton's method and we call these methods Newton-like methods. A prototype algorithm of Newton-like methods for solving problem [UMP] is given by the following iterative scheme:

\section{Algorithm [NL]}

Step 0. Given an initial guess $x_{0} \in R^{n}$ and an initial matrix $B_{0} \in R^{n \times n}$. Set $k=0$.

Step 1. Convergence check.

Step 2. Obtain a search direction $d_{k}$ by solving the linear system of equations

$$
B_{k} d=-\nabla f\left(x_{k}\right) \text {. }
$$

Step 3. Set $x_{k+1}=x_{k}+d_{k}$.

Step 4. Construct a new matrix $B_{k+1}$, and set $k:=k+1$. Go to Step 1 .

Here a matrix $B_{k}$ is an approximation to the Hessian matrix $\nabla^{2} f\left(x_{k}\right)$ or itself. Step 4 characterizes this method and, by varying a choice of $B_{k}$, Algorithm [NL] gives various kinds of methods. For example, we have Newton's method by setting $B_{k}=\nabla^{2} f\left(x_{k}\right)$, and we have secant methods by updating a current matrix $B_{k}$ to obtain a new matrix $B_{k+1}$. In case of secant methods, the new matrix $B_{k+1}$ is given by

$$
B_{k+1}=B_{k}+\Delta B_{k}
$$


where $\Delta B_{k}$ is a correction matrix, and the matrix is imposed on satisfying the secant condition

$$
B_{k+1} s_{k}=y_{k}
$$

where

$$
s_{k}=x_{k+1}-x_{k} \quad \text { and } \quad y_{k}=\nabla f\left(x_{k+1}\right)-\nabla f\left(x_{k}\right) .
$$

This condition comes from Taylor expansion of $f(x)$ such that

$$
\nabla f\left(x_{k}\right) \approx \nabla f\left(x_{k+1}\right)+\nabla^{2} f\left(x_{k+1}\right)\left(x_{k}-x_{k+1}\right) \text {. }
$$

Information that is used in determining $B_{k+1}$ is contained in vectors $s_{k}$ and $y_{k}$. One requires that $B_{k+1} s_{k}$ be a good approximation to $\nabla^{2} f\left(x_{k+1}\right) s_{k}$ or $\nabla^{2} f\left(x_{*}\right) s_{k}$.

Since the Hessian matrix is symmetric, it is desirable for $B_{k}$ to be symmetric. Within the framework of the line search globalization strategy, which finds a step size $\alpha_{k}$ such that $f\left(x_{k}+\alpha_{k} d_{k}\right)<f\left(x_{k}\right)$ and sets $x_{k+1}=x_{k}+\alpha_{k} d_{k}$, the positive definiteness of $B_{k}$ guarantees that a direction $d_{k}$ generated in Step 2 becomes a descent search direction for $f(x)$. In addition, a good information about the Hessian matrix of the objective function enables us to obtain a Newton-like method that possesses a rapid convergence property. Therefore, it is very important to construct a symmetric positive definite matrix $B_{k+1}$ that satisfies some condition with Hessian information, such as the secant condition (1.2). For this purpose, in general, we consider a matrix $B_{+} \in R^{n \times n}$ that satisfies the following condition.

\section{[SSP-Condition]}

(1) Secant condition: Given vectors $s, z \in R^{n}$ such that $s^{T} z>0, B_{+}$satisfies the condition

$$
B_{+} s=z
$$

(2) Symmetry: $B_{+}$is symmetric.

(3) Positive definiteness: $B_{+}$is positive definite.

A matrix $B_{+}$that satisfies the SSP-condition is called the SSP-matrix, and a matrix $N_{+} \in R^{m \times n}(m \geq n)$ that satisfies $N_{+}^{T} N_{+}=B_{+}$is called the factorized form of $B_{+}$. When $B_{k}$ is an SSP-matrix in Algorithm [NL], we call the algorithm the symmetric positive definite class of Newton-like methods. Note that the positivity of $s^{T} z$ is a necessary condition for the existence of the SSP-matrix. In Secant condition (1.4), it is desiable to choose $z$ as a good approximation to $\nabla^{2} f\left(x_{*}\right) s$, and we can choose such a vector $z$ based on a structure of the problem. In the case of secant methods, we usually set $s=s_{k}$ and $z=y_{k}$ for $s_{k}$ and $y_{k}$ in (1.3) and condition (1.4) reduces to condition (1.2).

Secant methods which preserve the positive definiteness have been studied. For example, Gay [9] dealt with the rank-two updates. Brodlie et al. [2] proposed the real product form. Factorized versions of the updates were studied, for example, by Goldfarb [10], Dennis and Schnabel [6], Yabe [14], Yamaki and Yabe [15], and Ip [12]. Dennis and Schnabel, and Ip derived the factorized updates by using least change secant updates. Furthermore, Zhang and Tewarson [16] discussed the Cholesky factorization of the updating formulae. In this paper, we will deal with a wider class of positive definite matrices than updating formulae in secant methods.

The objects of this paper are showing a general form and its factorized form of SSPmatrices to propose a symmetric positive definite class of Newton-like methods, and applying it to secant methods and other methods. By finding such a general form, it may be 
expected that we can unify Newton-like methods that satisfy the SSP-condition, and discuss convergence properties of methods from the viewpoint of the SSP-matrix.

The present paper is organized as follows. In Section 2, we will give a general form and its factorized form that satisfy the SSP-condition. We will also derive a bounded deterioration property for the general form, which is very useful to show convergence properties of specific numerical methods given in the subsequent section. In Section 3, we will propose a symmetric positive definite class of secant methods as an application of the SSP-matrix and will discuss its convergence property. Specifically, in Subsection 3.1, we will derive a new family of secant methods and in Subsection 3.2, show local and q-superlinear convergence of our method. Furthermore, in Subsection 3.3, the Broyden family will be described as a subfamily of our new family. In Section 4, we will propose a modification of the Gauss-Newton method for nonlinear least squares problems as another application of the SSP-matrix.

Throughout this paper, for a vector, \|\| denotes the $l_{2}$ norm and for a matrix, \|\| denotes the operator norm induced from the $l_{2}$ vector norm. For a matrix $Q \in R^{n \times n}$ and a symmetric nonsingular matrix $M \in R^{n \times n}$, let $\|Q\|_{F}$ denote the Frobenius norm of $Q$ and let $\|Q\|_{F, M}$ be $\left\|M^{-1} Q M^{-1}\right\|_{F}$. By the equivalence of norms, there exists a positive constant $\eta$ such that

$$
\frac{1}{\eta}\|Q\|_{F, M} \leq\|Q\| \leq \eta\|Q\|_{F, M}
$$

For a symmetric positive definite matrix $S \in R^{n \times n}$, let $S^{\frac{1}{2}}$ denote a symmetric matrix such that $\left(S^{\frac{1}{2}}\right)^{2}=S$.

\section{General Form of an SSP-matrix}

In this section, we will present a general form and its factorized form of SSP-matrices. Both the forms will be useful tools for studying Newton-like methods. First we give a general form of SSP-matrices.

Theorem 1 A general form of SSP-matrices is given by the form

$$
B_{+}=\dot{C}-\frac{C s s^{T} C}{s^{T} C s}+\frac{z z^{T}}{s^{T} z}+\left(s^{T} C s\right) w w^{T}
$$

or equivalently,

$$
B_{+}=\left(I-\frac{s u^{T} \Phi}{s^{T} \Phi^{T} u}\right)^{T} \Phi^{T} \Phi\left(I-\frac{s u^{T} \Phi}{s^{T} \Phi^{T} u}\right)+\frac{z z^{T}}{s^{T} z}
$$

with

$$
w=\frac{\Phi^{T} u}{s^{T} \Phi^{T} u}-\frac{C s}{s^{T} C s} \quad \text { and } \quad C=\Phi^{T} \Phi,
$$

where $\Phi$ is any full column rank matrix, and $u$ is any vector such that $s^{T} \Phi^{T} u \neq 0$.

Proof. It is easily shown that $B_{+}$satisfies the secant condition. Moreover, setting $\gamma=$ $\left(p^{T} C p\right)\left(s^{T} C s\right)-\left(p^{T} C s\right)^{2}$ for any nonzero vector $p \in R^{n}$, we have

$$
p^{T} B_{+} p=\frac{\gamma}{s^{T} C s}+\frac{\left(p^{T} z\right)^{2}}{s^{T} z}+\left(s^{T} C s\right)\left(p^{T} w\right)^{2} .
$$

Since Cauchy-Schwarz inequality implies $\gamma \geq 0$, we at least have $p^{T} B_{+} p \geq 0$. If $\gamma>0$, then $p^{T} B_{+} p>0$. If $\gamma=0$, then $p=\tau s$ for some $\tau \neq 0$ and $\left(p^{T} z\right)^{2}=\left(\tau s^{T} z\right)^{2}>0$, so we have $p^{T} B_{+} p>0$. This implies that $B_{+}$is positive definite. Thus $B_{+}$in (2.1) (or (2.2)) satisfies the SSP-condition. 
Conversely, let $\tilde{B}_{+}$be any matrix that satisfies the SSP-condition. Since $\tilde{B}_{+}$is symmetric positive definite, there exists a full column rank matrix $\tilde{F}_{+} \in R^{m \times n}$ such that $\tilde{B}_{+}=\tilde{F}_{+}^{T} \tilde{F}_{+}$. Setting $\Phi=\tilde{F}_{+}$yields $C=\tilde{B}_{+}$. By choosing $u$ such that $\Phi^{T} u=z$, we have $w=0$. Then the righthand side in (2.1) yields

$$
C-\frac{C s s^{T} C}{s^{T} C s}+\frac{z z^{T}}{s^{T} z}+\left(s^{T} C s\right) w w^{T}=\tilde{B}_{+}-\frac{\tilde{B}_{+} s s^{T} \tilde{B}_{+}}{s^{T} \tilde{B}_{+} s}+\frac{z z^{T}}{s^{T} z}=\tilde{B}_{+} .
$$

This implies that $\tilde{B}_{+}$can be formed as $(2.1)$.

Hence the matrix (2.1) is a general form of SSP-matrices.

Though an expression of the general form is not unique and there exist various types of forms, expression (2.1) is convenient to us in analyzing convergence properties of methods proposed below.

Since the SSP-matrix is symmetric positive definite, it can be represented by a factorized form. It is meaningful to construct a factorized form of SSP-matrices. Then following Yamaki and Yabe [15], we obtain the next theorem.

Theorem 2 The matrix $N_{+}$given by

$$
\begin{aligned}
N_{+} & =\Phi\left(I-\frac{s u^{T} \Phi}{s^{T} \Phi^{T} u}\right)+\frac{u z^{T}}{\sqrt{s^{T} z}\|u\|} \\
& =\Phi-\frac{\Phi s u^{T} \Phi}{s^{T} \Phi^{T} u}+\frac{u z^{T}}{\sqrt{s^{T} z}\|u\|}
\end{aligned}
$$

is a factorized form of the general form $B_{+}$in (2.1).

Proof. It follows from a direct calculation that $N_{+}^{T} N_{+}=B_{+}$.

Note that a factorized form is not unique for the matrix (2.1). In fact, for any orthogonal matrix $\Gamma,\left(\Gamma N_{+}\right)^{T}\left(\Gamma N_{+}\right)$also yields $B_{+}$of $(2.1)$. Thus if $N_{+}$with $\Phi$ and $u$ is a factorized form of (2.1), then so is $\Gamma N_{+}$.

Now we derive a bounded deterioration property for the general form (2.1). Broyden, Dennis and Moré [3] originally proposed the bounded deterioration principle for secant methods, and our result is an application of the bounded deterioration property to SSPmatrices. This plays an important role in proving of convergence theorems of a method proposed in Section 3. Let $M \in R^{n \times n}$ be a symmetric nonsingular matrix. Set

$$
\widehat{C}=M^{-1} C M^{-1}, \quad \widehat{z}=M^{-1} z, \quad \widehat{s}=M s \quad \text { and } \quad \widehat{B}_{+}=M^{-1} B_{+} M^{-1} .
$$

We note that under the above relations, $\widehat{B}_{+}$also satisfies the secant condition with respect to $\widehat{s}, \widehat{z}$, and that $s^{T} z=\widehat{s}^{T} \widehat{z}$ holds.

Then the bounded deterioration property for the general form (2.1) is given by the following theorem. In this theorem, equation (2.10) means the bounded deterioration property, and a positive scalar $\sigma^{\sharp}$ is a significant factor and plays an important role in showing the fast convergence property given later on (see for example the proofs of Theorems 4 and 5 ).

Theorem 3 Suppose that, for $s$ and $z$, there exist positive constants $\zeta, \beta_{1}, \beta_{2}, \gamma_{1}, \gamma_{2}$ and a positive scalar $\sigma^{\sharp}$ such that

$$
\|\widehat{z}-\widehat{s}\| \leq \zeta \sigma^{\sharp}\|\widehat{s}\|
$$


and

$$
\beta_{1}\|\hat{s}\|^{2} \leq s^{T} z \leq \beta_{2}\|\hat{s}\|^{2}, \quad \gamma_{1}\|\widehat{s}\| \leq\|\hat{z}\| \leq \gamma_{2}\|\widehat{s}\| .
$$

Assume that, for some positive constants $\delta^{\prime}$ and $K^{\prime}$,

$$
\|\widehat{C}-I\|_{F} \leq \delta^{\prime} \quad \text { and } \quad\|\widehat{C}\| \leq K^{\prime} .
$$

Let the matrix $B_{+}$be generated by (2.1). Assume that the vector $u$ is chosen such that $w$ in (2.3) satisfies

$$
\left\|\left(s^{T} C s\right)\left(w w^{T}-v v^{T}\right)\right\|_{F, M} \leq \rho_{1} \frac{\|(\widehat{C}-I) \widehat{s}\|^{2}}{\|\widehat{s}\|^{2}}+\rho_{2} \sigma^{\sharp}
$$

for positive constants $\rho_{1}$ and $\rho_{2}$, where

$$
v=\frac{z}{s^{T} z}-\frac{C s}{s^{T} C s} .
$$

Then

$$
\begin{aligned}
\left\|B_{+}-M^{2}\right\|_{F, M} \leq \| C- & M^{2} \|_{F, M}+\mu \sigma^{\sharp} \\
& +\left(\rho_{1}-\frac{1}{2 \delta^{\prime}}\right) \frac{\|(\widehat{C}-I) \widehat{s}\|^{2}}{\|\widehat{s}\|^{2}}
\end{aligned}
$$

where

$$
\mu=\rho_{2}+\zeta\left(\frac{4 K^{\prime}}{\beta_{1}}\left(\frac{\zeta}{\beta_{1}}+1\right)+\frac{\gamma_{2}^{2}}{\beta_{1}}+\gamma_{2}+1\right) .
$$

Proof. We prove this theorem in a similar way to the proof of Lemma 3.5 in [13], which showed the bounded deterioration property for the Broyden family of the secant method. Note that the general form (2.1) can be represented by

$$
B_{+}=A+\left(s^{T} C s\right)\left(w w^{T}-v v^{T}\right)
$$

where

$$
A=\left(I-\frac{s z^{T}}{s^{T} z}\right)^{T} C\left(I-\frac{s z^{T}}{s^{T} z}\right)+\frac{z z^{T}}{s^{T} z} .
$$

First we will give an estimate for the matrix $A$. It follows from (2.5) and (2.12) that

$$
M^{-1}\left(A-M^{2}\right) M^{-1}=D+E+E^{T}+F+G,
$$

where

$$
\begin{aligned}
D & =\left(I-\frac{\widehat{s} \widehat{s}^{T}}{\|\widehat{s}\|^{2}}\right)(\widehat{C}-I)\left(I-\frac{\widehat{s} \widehat{s}^{T}}{\|\widehat{s}\|^{2}}\right) \\
E & =\left(I-\frac{\widehat{s} \widehat{s}^{T}}{\|\widehat{s}\|^{2}}\right) \widehat{C}\left(\frac{\widehat{s} \widehat{s}^{T}}{\|\widehat{s}\|^{2}}-\frac{\widehat{s} \widehat{z}^{T}}{\widehat{s}^{T} \widehat{z}}\right), \\
F & =\left(\frac{\widehat{s} \widehat{s}^{T}}{\|\widehat{s}\|^{2}}-\frac{\widehat{s} \widehat{z}^{T}}{\widehat{s}^{T} \widehat{z}}\right)^{T} \widehat{C}\left(\frac{\widehat{s} \widehat{s}^{T}}{\|\widehat{s}\|^{2}}-\frac{\widehat{s} \widehat{z}^{T}}{\widehat{s}^{T} \widehat{z}}\right), \\
G & =\frac{\widehat{z} \widehat{z}^{T}}{\widehat{s}^{T} \widehat{z}}-\frac{\widehat{s} \widehat{s}^{T}}{\|\widehat{s}\|^{2}} .
\end{aligned}
$$


If $C \neq M^{2}$, then we have

$$
\begin{aligned}
\|D\|_{F}^{2} & \leq\left\|I-\frac{\widehat{s} \widehat{s}^{T}}{\|\widehat{s}\|^{2}}\right\|^{2}\left\|(\widehat{C}-I)\left(I-\frac{\widehat{s} \widehat{s}^{T}}{\|\widehat{s}\|^{2}}\right)\right\|_{F}^{2} \\
& =\left\|(\widehat{C}-I)\left(I-\frac{\widehat{s} \widehat{s}^{T}}{\|\widehat{s}\|^{2}}\right)\right\|_{F}^{2} \\
& =\|\widehat{C}-I\|_{F}^{2}-\frac{\|(\widehat{C}-I) \widehat{s}\|^{2}}{\|\widehat{s}\|^{2}} \\
& =\left(\|\widehat{C}-I\|_{F}-\frac{1}{2} \frac{\|(\widehat{C}-I) \widehat{s}\|^{2}}{\|\widehat{C}-I\|_{F}\|\widehat{s}\|^{2}}\right)^{2}-\left(\frac{1}{2} \frac{\|(\widehat{C}-I) \widehat{s}\|^{2}}{\|\widehat{C}-I\|_{F}\|\widehat{s}\|^{2}}\right)^{2} \\
& \leq\left(\|\widehat{C}-I\|_{F}-\frac{1}{2} \frac{\|(\widehat{C}-I) \widehat{s}\|^{2}}{\|\widehat{C}-I\|_{F}\|\widehat{s}\|^{2}}\right)^{2}
\end{aligned}
$$

and

$$
\begin{aligned}
\|\widehat{C}-I\|_{F}-\frac{1}{2} \frac{\|(\widehat{C}-I) \widehat{s}\|^{2}}{\|\widehat{C}-I\|_{F}\|\widehat{s}\|^{2}} & \geq\|\widehat{C}-I\|_{F}-\frac{1}{2} \frac{\|\widehat{C}-I\|_{F}^{2}\|\widehat{s}\|^{2}}{\|\widehat{C}-I\|_{F}\|\widehat{s}\|^{2}} \\
& =\frac{1}{2}\|\widehat{C}-I\|_{F}>0 .
\end{aligned}
$$

Thus by (2.8), (2.13) and (2.14), we have

$$
\|D\|_{F} \leq\left\|C-M^{2}\right\|_{F, M}-\frac{1}{2 \delta^{\prime}} \frac{\|(\widehat{C}-I) \widehat{s}\|^{2}}{\|\hat{s}\|^{2}} .
$$

If $C=M^{2}$, then the above inequality also holds, because $D$ becomes the zero matrix. Furthermore, since the assumptions yield

$$
\begin{aligned}
\left\|\frac{\widehat{s} \widehat{s}^{T}}{\widehat{s}^{T} \widehat{s}}-\frac{\widehat{s} \widehat{z}^{T}}{\widehat{s}^{T} \widehat{z}}\right\|_{F} & =\left\|\frac{\widehat{s}^{T}(\widehat{z}-\widehat{s})}{\|\widehat{s}\|^{2} \widehat{s}^{T} \widehat{z}} \widehat{s}^{T}+\frac{\widehat{s}(\widehat{s}-\widehat{z})^{T}}{\widehat{s}^{T} \widehat{z}}\right\|_{F} \\
& \leq \frac{\|\widehat{s}\|^{3}\|\widehat{z}-\widehat{s}\|}{\|\widehat{s}\|^{2} \widehat{s}^{T} \widehat{z}}+\frac{\|\widehat{s}\|\|\widehat{z}-\widehat{s}\|}{\widehat{s}^{T} \widehat{z}} \\
& =2 \frac{\|\widehat{s}\|\|\widehat{z}-\widehat{s}\|}{\widehat{s}^{T} \widehat{z}} \\
& \leq \frac{2 \zeta}{\beta_{1}} \sigma^{\sharp}
\end{aligned}
$$

and

$$
\begin{aligned}
\left\|\frac{\widehat{z} \widehat{z}^{T}}{\widehat{s}^{T} \widehat{z}}-\frac{\widehat{s} \widehat{s}^{T}}{\widehat{s}^{T} \widehat{s}}\right\|_{F} & =\left\|\left(\frac{\widehat{z} \widehat{z}^{T}}{\widehat{s}^{T} \widehat{z}}-\frac{\widehat{z} \widehat{z}^{T}}{\widehat{s}^{T} \widehat{s}}\right)+\left(\frac{\widehat{z} \widehat{z}^{T}}{\widehat{s}^{T} \widehat{s}}-\frac{\widehat{z} \widehat{s}^{T}}{\widehat{s}^{T} \widehat{s}}\right)+\left(\frac{\widehat{z} \widehat{s}^{T}}{\widehat{s}^{T} \widehat{s}}-\frac{\widehat{s} \widehat{s}^{T}}{\widehat{s}^{T} \widehat{s}}\right)\right\|_{F} \\
& \leq \frac{\|\hat{s}\|\|\widehat{z}\|^{2}\|\widehat{z}-\widehat{s}\|}{\widehat{s}^{T} \widehat{z}\|\widehat{s}\|^{2}}+\frac{\|\widehat{z}\|\|\widehat{z}-\widehat{s}\|}{\|\widehat{s}\|^{2}}+\frac{\|\widehat{s}\|\|\widehat{z}-\widehat{s}\|}{\|\widehat{s}\|^{2}} \\
& =\left(\frac{\|\widehat{z}\|^{2}}{\widehat{s}^{T} \widehat{z}}+\frac{\|\widehat{z}\|}{\|\widehat{s}\|}+1\right) \frac{\|\widehat{z}-\widehat{s}\|}{\|\widehat{s}\|} \\
& \leq\left(\frac{\gamma_{2}^{2}}{\beta_{1}}+\gamma_{2}+1\right) \zeta \sigma^{\sharp},
\end{aligned}
$$


we see that

$$
\begin{aligned}
\|E\|_{F} & \leq\left\|I-\frac{\widehat{s} \widehat{s}^{T}}{\|\widehat{s}\|^{2}}\right\|\|\widehat{C}\|\left\|\frac{\widehat{s} \widehat{s}^{T}}{\|\widehat{s}\|^{2}}-\frac{\widehat{s} \widehat{z}^{T}}{\widehat{s}^{T} \widehat{z}}\right\|_{F} \leq K^{\prime} \frac{2 \zeta}{\beta_{1}} \sigma^{\sharp} \\
\|F\|_{F} & \leq\|\widehat{C}\|\left\|\frac{\widehat{s} \widehat{s}^{T}}{\|\widehat{s}\|^{2}}-\frac{\widehat{s} \widehat{z}^{T}}{\widehat{s}^{T} \widehat{z}}\right\|_{F}^{2} \leq K^{\prime}\left(\frac{2 \zeta}{\beta_{1}} \sigma^{\sharp}\right)^{2}, \\
\|G\|_{F} & \leq\left(\frac{\gamma_{2}^{2}}{\beta_{1}}+\gamma_{2}+1\right) \zeta \sigma^{\sharp} .
\end{aligned}
$$

Thus the relation

$$
\left\|A-M^{2}\right\|_{F, M} \leq\|D\|_{F}+2\|E\|_{F}+\|F\|_{F}+\|G\|_{F}
$$

implies

$$
\left\|A-M^{2}\right\|_{F, M} \leq\left\|C-M^{2}\right\|_{F, M}-\frac{\|(\widehat{C}-I) \widehat{s}\|^{2}}{2 \delta^{\prime}\|\widehat{s}\|^{2}}+\mu^{\prime} \sigma^{\sharp}
$$

where

$$
\mu^{\prime}=\zeta\left(\frac{4 K^{\prime}}{\beta_{1}}+\frac{4 K^{\prime} \zeta}{\beta_{1}^{2}}+\frac{\gamma_{2}^{2}}{\beta_{1}}+\gamma_{2}+1\right)
$$

Therefore, it follows from equations (2.9), (2.11) and (2.15) that

$$
\left\|B_{+}-M^{2}\right\|_{F, M} \leq\left\|C-M^{2}\right\|_{F, M}+\mu \sigma^{\sharp}+\left(\rho_{1}-\frac{1}{2 \delta^{\prime}}\right) \frac{\|(\widehat{C}-I) \widehat{s}\|^{2}}{\|\widehat{s}\|^{2}},
$$

where $\mu=\rho_{2}+\mu^{\prime}$.

To end this section, we derive a general form of a matrix $H_{+}$that corresponds to $B_{+}^{-1}$. In this case, the first condition of the SSP-condition is replaced by

$$
H_{+} z=s .
$$

Then in a similar way to the preceding, we obtain a general form for $H_{+}$and its factorized form $\tilde{N}_{+}$that satisfy the SSP-condition as follows:

$$
H_{+}=\tilde{C}-\frac{\tilde{C} z z^{T} \tilde{C}}{z^{T} \tilde{C} z}+\frac{s s^{T}}{z^{T} s}+\left(z^{T} \tilde{C} z\right) \tilde{w} \tilde{w}^{T}
$$

or equivalently,

$$
H_{+}=\left(I-\frac{z \tilde{u}^{T} \tilde{\Phi}}{z^{T} \tilde{\Phi}^{T} \tilde{u}}\right)^{T} \tilde{C}\left(I-\frac{z \tilde{u}^{T} \tilde{\Phi}}{z^{T} \tilde{\Phi}^{T} \tilde{u}}\right)+\frac{s s^{T}}{z^{T} s}
$$

with

$$
\tilde{w}=\frac{\tilde{\Phi}^{T} \tilde{u}}{z^{T} \tilde{\Phi}^{T} \tilde{u}}-\frac{\tilde{C} z}{z^{T} \tilde{C} z}, \quad \tilde{C}=\tilde{\Phi}^{T} \tilde{\Phi}
$$

and

$$
\tilde{N}_{+}=\tilde{\Phi}\left(I-\frac{z \tilde{u}^{T} \tilde{\Phi}}{z^{T} \tilde{\Phi}^{T} \tilde{u}}\right)+\frac{\tilde{u} s^{T}}{\sqrt{z^{T} s}\|\tilde{u}\|}=\tilde{\Phi}-\frac{\tilde{\Phi} z \tilde{u}^{T} \tilde{\Phi}}{z^{T} \tilde{\Phi}^{T} \tilde{u}}+\frac{\tilde{u} s^{T}}{\sqrt{z^{T} s}\|\tilde{u}\|}
$$

where $\tilde{\Phi}$ is any full column rank matrix and $\tilde{u}$ is any vector such that $z^{T} \tilde{\Phi}^{T} \tilde{u} \neq 0$. By using this form, we can obtain a search direction

$$
d_{k}=-H_{k} \nabla f\left(x_{k}\right)
$$

without solving the linear system of equations (1.1) in Algorithm [NL]. 


\section{Application of SSP-matrix to Secant Methods}

\subsection{A new family of secant updates}

In this section, we consider the secant method for solving the unconstrained minimization problem [UMP]. The method is characterized by the fact that a new Hessian approximation $B_{k+1}$ in Step 4 of Algorithm [NL] is generated by updating a current matrix $B_{k}$ so as to satisfy the secant condition (1.2). By using the general forms (2.1) and (2.4), we obtain a new family and its factorized form for $B_{k}$ that satisfy the SSP-condition.

In what follows, we assume that $\nabla^{2} f\left(x_{*}\right)$ is symmetric positive definite and we define

$$
M=\nabla^{2} f\left(x_{*}\right)^{\frac{1}{2}} \text {. }
$$

By setting $s=s_{k}, z=y_{k}, \Phi=L_{k} \in R^{n \times n}$ and $C=B_{k}=L_{k}^{T} L_{k}$ in (2.1) and (2.4), we obtain $B_{+}=B_{k+1}$ and $N_{+}=L_{k+1}$ as follows:

$$
B_{k+1}=B_{k}-\frac{B_{k} s_{k} s_{k}^{T} B_{k}}{s_{k}^{T} B_{k} s_{k}}+\frac{y_{k} y_{k}^{T}}{s_{k}^{T} y_{k}}+\left(s_{k}^{T} B_{k} s_{k}\right) w_{k} w_{k}^{T},
$$

and

$$
L_{k+1}=L_{k}-\frac{L_{k} s_{k} u_{k}^{T} L_{k}}{s_{k}^{T} L_{k}^{T} u_{k}}+\frac{u_{k} y_{k}^{T}}{\sqrt{s_{k}^{T} y_{k}}\left\|u_{k}\right\|},
$$

where $u_{k}$ is any vector such that $s_{k}^{T} L_{k}^{T} u_{k} \neq 0$ and

$$
w_{k}=\frac{L_{k}^{T} u_{k}}{s_{k}^{T} L_{k}^{T} u_{k}}-\frac{B_{k} s_{k}}{s_{k}^{T} B_{k} s_{k}} .
$$

The form (3.2) is a class of symmetric positive definite secant updates that includes the parameter vector $u_{k} \in R^{n}$, and the form (3.3) is its factorized form. Note that (3.2) is a symmetric positive definite update with an at most rank-three correction, while (3.3) is an update with an at most rank-two correction. A specific choice of $u_{k}$ yields a symmetric positive definite update with a rank-two or rank-three correction. For example, a rank-one update in (3.3) yields a rank-two update in (3.2), and a rank-two update in (3.3) yields a rank-three update in (3.2), which are presented below. A unified approach of symmetric positive definite updates with a rank-two correction can be found in [9].

\subsection{Local and q-superlinear convergence}

Our objective in this subsection is to show local and q-superlinear convergence of the secant method with (3.2) and (3.3). We first prove that this family satisfies the bounded deterioration property given by Broyden, Dennis and Moré [3]. Next, q-superlinear convergence of our method follows from the Dennis-Moré characterization [5]. For this purpose, we will use Theorem 3 given in Section 2.

Let $D$ be an open convex subset of $R^{n}$, which contains a local minimizer $x_{*}$ of $f(x)$. We assume the following standard conditions.

(A1) There exists a positive constant $\xi$ such that

$$
\left\|\nabla^{2} f(x)-\nabla^{2} f\left(x^{\prime}\right)\right\| \leq \xi\left\|x-x^{\prime}\right\|
$$

for any $x, x^{\prime}$ in $D$.

(A2) $\nabla^{2} f$ is symmetric positive definite at $x_{*}$. 
It follows easily from assumption (A1) and the mean value theorem that, for any $x, x^{\prime} \in D$,

$$
\left\|\nabla f(x)-\nabla f\left(x^{\prime}\right)-\nabla^{2} f\left(x_{*}\right)\left(x-x^{\prime}\right)\right\| \leq \xi \max \left(\left\|x-x_{*}\right\|,\left\|x^{\prime}-x_{*}\right\|\right)\left\|x-x^{\prime}\right\|
$$

(see for example [7]). Define

$$
\sigma_{k}=\max \left(\left\|x_{k+1}-x_{*}\right\|,\left\|x_{k}-x_{*}\right\|\right) .
$$

For the matrix $M$ in (3.1), set

$$
\widehat{B}_{k}=M^{-1} B_{k} M^{-1}, \quad \widehat{y}_{k}=M^{-1} y_{k}, \quad \widehat{s}_{k}=M s_{k} \quad \text { and } \quad \widehat{B}_{k+1}=M^{-1} B_{k+1} M^{-1} .
$$

In the lemma below, we offer that conditions corresponding to (2.6) and (2.7) are satisfied. Lemma 1 Suppose that assumptions (A1) and (A2) hold. Then there exists a positive constant $\zeta$ such that

$$
\left\|\widehat{y}_{k}-\widehat{s}_{k}\right\| \leq \zeta \sigma_{k}\left\|\widehat{s}_{k}\right\|
$$

for any $x_{k}, x_{k+1} \in D$. Furthermore, there exist positive constants $\beta_{1}, \beta_{2}, \gamma_{1}$ and $\gamma_{2}$ such that

$$
\beta_{1}\left\|\widehat{s}_{k}\right\|^{2} \leq s_{k}^{T} y_{k} \leq \beta_{2}\left\|\widehat{s}_{k}\right\|^{2}
$$

and

$$
\gamma_{1}\left\|\widehat{s}_{k}\right\| \leq\left\|\widehat{y}_{k}\right\| \leq \gamma_{2}\left\|\widehat{s}_{k}\right\|
$$

for any $x_{k}$ and $x_{k+1}$ which satisfy

$$
\left\|x_{k}-x_{*}\right\| \leq \varepsilon \quad \text { and } \quad\left\|x_{k+1}-x_{*}\right\| \leq \varepsilon
$$

for $\varepsilon$ sufficiently small.

Proof. The results follow directly from Section 4 in [3].

We now establish the local and linear convergence theorem of our method.

Theorem 4 Suppose that assumptions (A1) and (A2) are satisfied. Let the matrix $B_{k}$ be updated by (3.2). Assume that the vector $u_{k}$ is chosen such that $w_{k}$ in (3.4) satisfies

$$
\left\|\left(s_{k}^{T} B_{k} s_{k}\right)\left(w_{k} w_{k}^{T}-v_{k} v_{k}^{T}\right)\right\|_{F, M} \leq \rho_{1} \frac{\left\|\left(\widehat{B}_{k}-I\right) \widehat{s}_{k}\right\|^{2}}{\left\|\widehat{s}_{k}\right\|^{2}}+\rho_{2} \sigma_{k}
$$

for positive constants $\rho_{1}$ and $\rho_{2}$ which are independent of $k$, where

$$
v_{k}=\frac{y_{k}}{s_{k}^{T} y_{k}}-\frac{B_{k} s_{k}}{s_{k}^{T} B_{k} s_{k}}
$$

Let the sequence $\left\{x_{k}\right\}$ be generated by

$$
x_{k+1}=x_{k}+s_{k} \quad \text { and } \quad B_{k} s_{k}=-\nabla f\left(x_{k}\right) .
$$

Then, for any $\nu \in(0,1)$, there exist positive constants $\varepsilon$ and $\delta$ such that if

$$
\left\|x_{0}-x_{*}\right\| \leq \varepsilon, \quad x_{0} \in D
$$

and

$$
\left\|B_{0}-\nabla^{2} f\left(x_{*}\right)\right\|_{F, M} \leq \delta
$$

the sequence $\left\{x_{k}\right\}$ is well defined and converges linearly to $x_{*}$ with

$$
\left\|x_{k+1}-x_{*}\right\| \leq \nu\left\|x_{k}-x_{*}\right\|
$$


Proof. Set

$$
\begin{gathered}
\mathcal{N}_{1}=\left\{x \in R^{n} \mid\left\|x-x_{*}\right\| \leq \varepsilon\right\} \\
\mathcal{N}_{2}=\left\{B \in R^{n \times n} \mid\left\|B-\nabla^{2} f\left(x_{*}\right)\right\|_{F, M} \leq 2 \delta\right\} .
\end{gathered}
$$

Choose $\varepsilon$ such that $\mathcal{N}_{1} \subset D$. Now we prove, by mathematical induction, that the following expressions $(\mathrm{E} 1 ; k)-(\mathrm{E} 3 ; k)$ hold for all $k \geq 0$ :

$$
B_{k} \in \mathcal{N}_{2}, \quad\left\|B_{k}\right\| \leq \tau_{1}, \quad \text { and } \quad\left\|B_{k}^{-1}\right\| \leq \tau_{2},
$$

$$
\begin{aligned}
&\left\|x_{k+1}-x_{*}\right\| \leq \nu\left\|x_{k}-x_{*}\right\|, \quad x_{k+1} \in \mathcal{N}_{1}, \\
&\left\|B_{k+1}-\nabla^{2} f\left(x_{*}\right)\right\|_{F, M} \leq\left\|B_{k}-\nabla^{2} f\left(x_{*}\right)\right\|_{F, M}+\mu \sigma_{k} \\
&+\left(\rho_{1}-\frac{1}{4 \delta}\right) \frac{\left\|\left(\widehat{B}_{k}-I\right) \widehat{s}_{k}\right\|^{2}}{\left\|\widehat{s}_{k}\right\|^{2}},
\end{aligned}
$$

where $\tau_{1}, \tau_{2}$ and $\mu$ are positive constants defined below.

We first consider the case of $k=0$.

$(\mathrm{E} 1 ; 0)$ The first and second results follow directly from the choice of the initial matrix. If we choose $\delta$ such that

$$
2 \eta\left\|\nabla^{2} f\left(x_{*}\right)^{-1}\right\| \delta \leq \frac{\nu}{1+\nu},
$$

the fact $B_{0} \in \mathcal{N}_{2}$ yields

$$
\left\|\nabla^{2} f\left(x_{*}\right)^{-1}\right\|\left\|B_{0}-\nabla^{2} f\left(x_{*}\right)\right\| \leq 2 \eta \delta\left\|\nabla^{2} f\left(x_{*}\right)^{-1}\right\| \leq \frac{\nu}{1+\nu}<1
$$

where $\eta$ is defined by (1.5). By the Banach perturbation lemma ([7]; Theorem 3.1.4), $B_{0}$ is nonsingular and

$$
\left\|B_{0}^{-1}\right\| \leq(1+\nu)\left\|\nabla^{2} f\left(x_{*}\right)^{-1}\right\|=\tau_{2} .
$$

$(\mathrm{E} 2 ; 0)$ It follows easily from $(3.5),(3.8)$ and $\nabla f\left(x_{*}\right)=0$ that

$$
\begin{aligned}
\left\|x_{1}-x_{*}\right\| & =\left\|x_{0}-B_{0}^{-1} \nabla f\left(x_{0}\right)-x_{*}\right\| \\
& =\left\|-B_{0}^{-1}\left(\nabla f\left(x_{0}\right)-\nabla f\left(x_{*}\right)-\nabla^{2} f\left(x_{*}\right)\left(x_{0}-x_{*}\right)-\left(B_{0}-\nabla^{2} f\left(x_{*}\right)\right)\left(x_{0}-x_{*}\right)\right)\right\| \\
& \leq\left\|B_{0}^{-1}\right\|\left(\left\|\nabla f\left(x_{0}\right)-\nabla f\left(x_{*}\right)-\nabla^{2} f\left(x_{*}\right)\left(x_{0}-x_{*}\right)\right\|+\left\|B_{0}-\nabla^{2} f\left(x_{*}\right)\right\|\left\|x_{0}-x_{*}\right\|\right) \\
& \leq \tau_{2}(\xi \varepsilon+2 \eta \delta)\left\|x_{0}-x_{*}\right\| .
\end{aligned}
$$

If we choose $\varepsilon$ and $\delta$ such that

$$
\tau_{2}(\xi \varepsilon+2 \eta \delta)<\nu
$$

we have

$$
\left\|x_{1}-x_{*}\right\| \leq \nu\left\|x_{0}-x_{*}\right\| \leq \varepsilon
$$

Thus $x_{1} \in \mathcal{N}_{1}$.

(E3;0) By setting

$$
s=s_{0}, \quad z=y_{0}, \quad \sigma^{\sharp}=\sigma_{0}, \quad \delta^{\prime}=2 \delta \quad \text { and } \quad K^{\prime}=\left\|M^{-1}\right\|^{2} \tau_{1},
$$

Theorem 3 and Lemma 1 yield

$$
\left\|B_{1}-\nabla^{2} f\left(x_{*}\right)\right\|_{F, M} \leq\left\|B_{0}-\nabla^{2} f\left(x_{*}\right)\right\|_{F, M}+\mu \sigma_{0}+\left(\rho_{1}-\frac{1}{4 \delta}\right) \frac{\left\|\left(\widehat{B}_{0}-I\right) \widehat{s}_{0}\right\|^{2}}{\left\|\widehat{s}_{0}\right\|^{2}} .
$$


Therefore the case of $k=0$ is proved.

We assume as an induction hypotheses that expressions $(\mathrm{E} 1 ; k)-(\mathrm{E} 3 ; k)$ hold for $k=$ $0, \ldots, t-1$. Then

$$
\left\|B_{k+1}-\nabla^{2} f\left(x_{*}\right)\right\|_{F, M} \leq\left\|B_{k}-\nabla^{2} f\left(x_{*}\right)\right\|_{F, M}+\mu \sigma_{k}+\left(\rho_{1}-\frac{1}{4 \delta}\right) \frac{\left\|\left(\widehat{B}_{k}-I\right) \widehat{s}_{k}\right\|^{2}}{\left\|\widehat{s}_{k}\right\|^{2}}
$$

for $k=0, \ldots, t-1$, and by summing both sides from $k=0$ to $t-1$, it follows that

$$
\left\|B_{t}-\nabla^{2} f\left(x_{*}\right)\right\|_{F, M} \leq\left\|B_{0}-\nabla^{2} f\left(x_{*}\right)\right\|_{F, M}+\mu \sum_{k=0}^{t-1} \sigma_{k}+\left(\rho_{1}-\frac{1}{4 \delta}\right) \sum_{k=0}^{t-1} \frac{\left\|\left(\widehat{B}_{k}-I\right) \widehat{s}_{k}\right\|^{2}}{\left\|\widehat{s}_{k}\right\|^{2}} .
$$

Note that, for $\sigma_{k}$ in (3.6),

$$
\sigma_{k}=\max \left(\left\|x_{k+1}-x_{*}\right\|,\left\|x_{k}-x_{*}\right\|\right)=\left\|x_{k}-x_{*}\right\| \leq \nu^{k} \varepsilon .
$$

If we choose $\varepsilon$ and $\delta$ such that

$$
\frac{\mu}{1-\nu} \varepsilon<\delta \quad \text { and } \quad \rho_{1}-\frac{1}{4 \delta}<0
$$

then we obtain

$$
\begin{aligned}
0 & \leq\left\|B_{t}-\nabla^{2} f\left(x_{*}\right)\right\|_{F, M} \\
& \leq\left\|B_{0}-\nabla^{2} f\left(x_{*}\right)\right\|_{F, M}+\mu \varepsilon \sum_{k=0}^{t-1} \nu^{k}+\left(\rho_{1}-\frac{1}{4 \delta}\right) \sum_{k=0}^{t-1} \frac{\left\|\left(\widehat{B}_{k}-I\right) \widehat{s}_{k}\right\|^{2}}{\left\|\widehat{s}_{k}\right\|^{2}} \\
& \leq\left\|B_{0}-\nabla^{2} f\left(x_{*}\right)\right\|_{F, M}+\frac{\mu}{1-\nu} \varepsilon+\left(\rho_{1}-\frac{1}{4 \delta}\right) \sum_{k=0}^{t-1} \frac{\left\|\left(\widehat{B}_{k}-I\right) \widehat{s}_{k}\right\|^{2}}{\left\|\widehat{s}_{k}\right\|^{2}} \\
& \leq\left\|B_{0}-\nabla^{2} f\left(x_{*}\right)\right\|_{F, M}+\frac{\mu}{1-\nu} \varepsilon \\
& \leq 2 \delta,
\end{aligned}
$$

which implies $B_{t} \in \mathcal{N}_{2}$. We can prove the rest of $(\mathrm{E} 1 ; t),(\mathrm{E} 2 ; t)$ and $(\mathrm{E} 3 ; t)$ in the same way as the case of $k=0$.

This concludes the induction, and the proof of the theorem.

The following theorem shows q-superlinear convergence of our method.

Theorem 5 Suppose that all conditions of Theorem 4 hold. Then the sequence $\left\{x_{k}\right\}$ generated by the scheme (3.8) with the family (3.2) converges q-superlinearly to $x_{*}$.

Proof. It follows directly from (3.9) in the proof of Theorem 4 that, for all $t \geq 1$,

$$
0 \leq\left(\frac{1}{4 \delta}-\rho_{1}\right) \sum_{k=0}^{t-1} \frac{\left\|\left(\widehat{B}_{k}-I\right) \widehat{s}_{k}\right\|^{2}}{\left\|\widehat{s}_{k}\right\|^{2}} \leq\left\|B_{0}-\nabla^{2} f\left(x_{*}\right)\right\|_{F, M}+\frac{\mu}{1-\nu} \varepsilon \leq 2 \delta .
$$

Since this guarantees the convergence of the infinite series

$$
\sum_{k=0}^{\infty} \frac{\left\|\left(\widehat{B}_{k}-I\right) \widehat{s}_{k}\right\|^{2}}{\left\|\widehat{s}_{k}\right\|^{2}}
$$

we have

$$
\lim _{k \rightarrow \infty} \frac{\left\|\left(\widehat{B}_{k}-I\right) \widehat{s}_{k}\right\|}{\left\|\widehat{s}_{k}\right\|}=0
$$


Thus the relation

$$
\frac{\left\|\left(B_{k}-\nabla^{2} f\left(x_{*}\right)\right) s_{k}\right\|}{\left\|s_{k}\right\|}=\frac{\left\|M\left(M^{-1} B_{k} M^{-1}-I\right) M s_{k}\right\|}{\left\|M s_{k}\right\|} \frac{\left\|M s_{k}\right\|}{\left\|s_{k}\right\|} \leq\|M\|^{2} \frac{\left\|\left(\widehat{B}_{k}-I\right) \widehat{s}_{k}\right\|}{\left\|\widehat{s}_{k}\right\|}
$$

implies

$$
\lim _{k \rightarrow \infty} \frac{\left\|\left(B_{k}-\nabla^{2} f\left(x_{*}\right)\right) s_{k}\right\|}{\left\|s_{k}\right\|}=0 .
$$

This is the necessary and sufficient condition that the sequence $\left\{x_{k}\right\}$ converges q-superlinearly to $x_{*}$ [5]. Therefore, the proof is complete.

\subsection{Example}

In this subsection, we introduce the symmetric positive definite class of Broyden family as a subfamily of our family (3.2) and give its convergence property as a corollary of Theorems 4 and 5 . For any scalar $\psi_{k}$, set

$$
u_{k}=\left(1-\psi_{k}\right) \frac{L_{k} s_{k}}{s_{k}^{T} B_{k} s_{k}}+\psi_{k} \frac{\left(L_{k}^{T}\right)^{-1} y_{k}}{s_{k}^{T} y_{k}} .
$$

Since this $u_{k}$ satisfies

$$
L_{k}^{T} u_{k}=\left(1-\psi_{k}\right) \frac{B_{k} s_{k}}{s_{k}^{T} B_{k} s_{k}}+\psi_{k} \frac{y_{k}}{s_{k}^{T} y_{k}} \quad \text { and } \quad s_{k}^{T} L_{k}^{T} u_{k}=1
$$

the vector $w_{k}$ in (3.4) becomes

$$
w_{k}=\psi_{k}\left(\frac{y_{k}}{s_{k}^{T} y_{k}}-\frac{B_{k} s_{k}}{s_{k}^{T} B_{k} s_{k}}\right) .
$$

Then from (3.2) and (3.3), we obtain the Broyden family and its factorized form as follows:

$$
B_{k+1}^{\text {Broyden }}=B_{k}-\frac{B_{k} s_{k} s_{k}^{T} B_{k}}{s_{k}^{T} B_{k} s_{k}}+\frac{y_{k} y_{k}^{T}}{s_{k}^{T} y_{k}}+\psi_{k}^{2}\left(s_{k}^{T} B_{k} s_{k}\right) v_{k} v_{k}^{T}
$$

with

$$
v_{k}=\frac{y_{k}}{s_{k}^{T} y_{k}}-\frac{B_{k} s_{k}}{s_{k}^{T} B_{k} s_{k}}
$$

and

$$
\begin{aligned}
L_{k+1}^{\text {Broyden }}=L_{k}+ & \left(1-\psi_{k}\right)\left(\frac{L_{k} s_{k}}{s_{k}^{T} B_{k} s_{k}}\right)\left(\sqrt{\lambda_{k}} y_{k}-B_{k} s_{k}\right)^{T} \\
& +\psi_{k} L_{k}\left(\sqrt{\lambda_{k}} B_{k}^{-1} y_{k}-s_{k}\right)\left(\frac{y_{k}}{s_{k}^{T} y_{k}}\right)^{T}
\end{aligned}
$$

where

$$
\lambda_{k}=\left[\left(1-\psi_{k}^{2}\right) \frac{s_{k}^{T} y_{k}}{s_{k}^{T} B_{k} s_{k}}+\psi_{k}^{2} \frac{y_{k}^{T} B_{k}^{-1} y_{k}}{s_{k}^{T} y_{k}}\right]^{-1} .
$$

In this case, by choosing specific parameter $\psi_{k}$ in the Broyden family, we have the BFGS update, the DFP update and their factorized forms as follows: 
BFGS update: Setting $\psi_{k}=0$ in $(3.11)-(3.13)$, we have

$$
B_{k+1}^{B F G S}=B_{k}-\frac{B_{k} s_{k} s_{k}^{T} B_{k}}{s_{k}^{T} B_{k} s_{k}}+\frac{y_{k} y_{k}^{T}}{s_{k}^{T} y_{k}}
$$

and

$$
L_{k+1}^{B F G S}=L_{k}+\left(\frac{L_{k} s_{k}}{s_{k}^{T} B_{k} s_{k}}\right)\left(\sqrt{\frac{s_{k}^{T} B_{k} s_{k}}{s_{k}^{T} y_{k}}} y_{k}-B_{k} s_{k}\right)^{T} .
$$

DFP update: Setting $\psi_{k}=1$ or $\psi_{k}=-1$ in $(3.11)-(3.13)$, we have

$$
B_{k+1}^{D F P}=B_{k}-\frac{B_{k} s_{k} y_{k}^{T}+y_{k} s_{k}^{T} B_{k}}{s_{k}^{T} y_{k}}+\left(1+\frac{s_{k}^{T} B_{k} s_{k}}{s_{k}^{T} y_{k}}\right) \frac{y_{k} y_{k}^{T}}{s_{k}^{T} y_{k}}
$$

and

$$
L_{k+1}^{D F^{\prime} P}=L_{k}+L_{k}\left(\sqrt{\frac{s_{k}^{T} y_{k}}{y_{k}^{T} B_{k}^{-1} y_{k}}} B_{k}^{-1} y_{k}-s_{k}\right)\left(\frac{y_{k}}{s_{k}^{T} y_{k}}\right)^{T} .
$$

Note that the forms (3.14) and (3.15) are identical with those given by Dennis and Schnabel [6].

Next, we give the local and q-superlinear convergence property of the Broyden family.

Corollary 1 Suppose that assumptions (A1) and (A2) are satisfied. Assume that there exists a positive constant $\psi^{\prime}$ such that $\left|\psi_{k}\right| \leq \psi^{\prime}$. Let the sequence $\left\{x_{k}\right\}$ be generated by (3.8). Then, the secant method with the Broyden family (3.11) has the local and q-superlinear convergence property.

Proof. Since $w_{k}=\psi_{k} v_{k}$, we see that

$\left\|\left(s_{k}^{T} B_{k} s_{k}\right)\left(w_{k} w_{k}^{T}-v_{k} v_{k}^{T}\right)\right\|_{F, M}=\left|\psi_{k}^{2}-1\right|\left\|\left(s_{k}^{T} B_{k} s_{k}\right) v_{k} v_{k}^{T}\right\|_{F, M} \leq\left(\left(\psi^{\prime}\right)^{2}+1\right)\left\|\left(s_{k}^{T} B_{k} s_{k}\right) v_{k} v_{k}^{T}\right\|_{F, M}$

In a similar way to the proof of Lemma 3.4 in [13], we can show that

$$
\left(\left(\psi^{\prime}\right)^{2}+1\right)\left\|\left(s_{k}^{T} B_{k} s_{k}\right) v_{k} v_{k}^{T}\right\|_{F, M} \leq \rho_{1} \frac{\left\|\left(\widehat{B}_{k}-I\right) \widehat{s}_{k}\right\|^{2}}{\left\|\widehat{s}_{k}\right\|^{2}}+\rho_{2} \sigma_{k}
$$

for $x_{k}, x_{k+1}$ very close to $x_{*}$, where $\rho_{1}$ and $\rho_{2}$ are positive constants. This implies that condition (3.7) holds. Therefore, by Theorems 4 and 5, the secant method with the Broyden family converges locally and q-superlinearly to $x_{*}$.

The preceding convergence result for the Broyden family corresponds to that shown by Stachurski [13].

\section{Concluding Remarks}

Within the framework of Newton-like methods for solving unconstrained minimization problems, we have obtained the general form (2.1) and its factorized form (2.4) of a matrix satisfying the SSP-condition. By finding such a general form, it may be expected that we can unify Newton-like methods that possess the positive definiteness and their convergence properties. Indeed Theorem 3 implies the bounded deterioration property for the SSP-matrix and has played an important role in proving the convergence properties of the methods proposed in Section 3. The standard bounded deterioration theorem deals with 
the case of $\sigma^{\sharp}=\sigma_{k}$, which is given in (3.6), for secant methods. In Section 3, we have derived a new family (3.2) of secant methods that contains the symmetric positive definite class of Broyden family (3.11), and we have shown local and q-superlinear convergence of our method.

To end this section, we will present two remarks. The first is a conjugate search direction for minimizing the strictly convex quadratic function. The second is a method which is not based on the updating scheme. This is a category of methods different from secant methods.

We first consider minimizing the strictly convex quadratic function

$$
f(x)=\frac{1}{2} x^{T} Q x+a^{T} x,
$$

where $Q \in R^{n \times n}$ is a symmetric positive definite matrix. It is known that if the secant method satisfies

$$
B_{k+1} s_{i}=y_{i}, \quad i=1, \ldots, k,
$$

then the method with the exact line search generates the $Q$-conjugate search directions $\left\{d_{k}\right\}$, i.e. $d_{i}^{T} Q d_{j}=0$ for $i \neq j$ (see [11]). Here the exact line search means that we choose a step size $\alpha_{k}$ such that $\nabla f\left(x_{k}+\alpha_{k} d_{k}\right)^{T} d_{k}=0$ holds. Consequently the minimum point can be obtained in at most $n$ iterations. We can discuss a condition under which the secant method with our family (3.2) generates $Q$-conjugate search directions. If we choose a parameter vector $u_{k}$ in (3.4) such that

$$
s_{k}^{T} L_{k}^{T} u_{k} \neq 0 \quad \text { and } \quad s_{j}^{T} L_{k}^{T} u_{k}=0, j=0,1, \ldots, k-1
$$

holds, then the secant method with (3.2) and the exact line search generates the $Q$-conjugate search directions $\left\{d_{k}\right\}$. Note that $s_{i}=\alpha_{i} d_{i}$ and $y_{i}=Q s_{i}$ hold at each iteration. We can show that if $B_{k} s_{i}=y_{i}, i=0, \ldots, k-1$, and $s_{i}^{T} Q s_{j}=0,0 \leq i<j \leq k$, are satisfied, then we have, for $i=0, \ldots, k$,

$$
B_{k+1} s_{i}=y_{i}
$$

and

$$
s_{k+1}^{T} Q s_{i}=s_{k+1}^{T} y_{i}=-\alpha_{k+1} \nabla f\left(x_{k+1}\right)^{T} B_{k+1}^{-1} y_{i}=-\alpha_{k+1} \nabla f\left(x_{k+1}\right)^{T} s_{i}=0,
$$

because $\nabla f\left(x_{k+1}\right)$ is orthogonal to the subspace spanned by $s_{0}, s_{1}, \ldots, s_{k}$. Therefore we obtain the result by mathematical induction. As a specific choice of $u_{k}$, the vector (3.10) satisfies condition (4.1). Thus the secant method with the Broyden family generates the $Q$-conjugate search directions under the exact line search (see [11]).

As shown in Section 3, secant methods are based on updating scheme and update the previous $B_{k}$ to obtain a new matrix $B_{k+1}$. However we does not restrict ourselves to the case. The SSP-matrix also involves methods which are not based on updating scheme. For example, we apply the SSP-matrix to the Gauss-Newton method and propose a modified method for nonlinear least squares problems:

$$
\text { minimize } \quad f(x)=\frac{1}{2} \sum_{j=1}^{m}\left(r_{j}(x)\right)^{2}, \quad n \leq m,
$$

where $r_{j}: R^{n} \rightarrow R, j=1,2, \ldots, m$, are nonlinear functions, and the residual vector is denoted by

$$
r(x)=\left(r_{1}(x), \ldots, r_{m}(x)\right)^{T} .
$$


Then the Hessian matrix of $f(x)$ is given as

$$
\nabla^{2} f(x)=J(x)^{T} J(x)+\sum_{j=1}^{m} r_{j}(x) \nabla^{2} r_{j}(x)
$$

where $J(x) \in R^{m \times n}$ denotes the Jacobian matrix of $r(x)$. In this case, the Hessian matrix $\nabla^{2} f(x)$ has a special structure, i.e. the first part $J(x)^{T} J(x)$ is available either analytically or from finite differences, but the second part $\sum_{j=1}^{m} r_{j}(x) \nabla^{2} r_{j}(x)$ is expensive to calculate. If we neglect the second part and set $B_{k}=J\left(x_{k}\right)^{T} J\left(x_{k}\right)$ in Algorithm [NL], we have the GaussNewton method. It is known that the Gauss-Newton method does not perform well for the large residual problems. The poor performance of the Gauss-Newton method is caused by the lack of information on the second part of the Hessian matrix. Hence it is expected that we may overcome this difficulty by applying the SSP-matrix to the Gauss-Newton method. Following Dennis [4], we consider the following secant condition for problem (4.2)

$$
B_{k} s_{k-1}=z_{k-1}
$$

with

$$
z_{k-1}=J\left(x_{k}\right)^{T} J\left(x_{k}\right) s_{k-1}+\left(J\left(x_{k}\right)-J\left(x_{k-1}\right)\right)^{T} r\left(x_{k}\right) .
$$

If the Jacobian matrix $J\left(x_{k}\right)$ is of full column rank, the matrix $J\left(x_{k}\right)^{T} J\left(x_{k}\right)$ is symmetric positive definite. Thus setting

$$
\Phi=J\left(x_{k}\right), \quad s=s_{k-1} \quad \text { and } \quad z=z_{k-1}
$$

in (2.1) and (2.4), we obtain a family $B_{k}$ and its factorized form $N_{k} \in R^{m \times n}$ as follows:

$$
B_{k}=J\left(x_{k}\right)^{T} J\left(x_{k}\right)-\frac{J\left(x_{k}\right)^{T} p_{k-1} p_{k-1}^{T} J\left(x_{k}\right)}{\left\|p_{k-1}\right\|^{2}}+\frac{z_{k-1} z_{k-1}^{T}}{s_{k-1}^{T} z_{k-1}}+\left\|p_{k-1}\right\|^{2} w_{k-1} w_{k-1}^{T}
$$

with

$$
w_{k-1}=\frac{J\left(x_{k}\right)^{T} u}{p_{k-1}^{T} u}-\frac{J\left(x_{k}\right)^{T} p_{k-1}}{\left\|p_{k-1}\right\|^{2}}
$$

and

$$
N_{k}=J\left(x_{k}\right)-\frac{p_{k-1} u^{T} J\left(x_{k}\right)}{p_{k-1}^{T} u}+\frac{u z_{k-1}^{T}}{\sqrt{s_{k-1}^{T} z_{k-1}}\|u\|}
$$

where $p_{k-1}=J\left(x_{k}\right) s_{k-1}$ and $u$ is any vector such that $p_{k-1}^{T} u \neq 0$. Since the family (4.4) - (4.6) contains information on the second part of the Hessian matrix in (4.3), it is a modification of the Gauss-Newton method. We should note that this is a method which is not based on the updating scheme. For a specific choice of $u$ as defined in (3.10), we have a family that corresponds to the Broyden family. This family contains the GN-BFGS and the GN-DFP methods proposed by Al-Baali and Fletcher [1] ( see also Dennis et al. [8]). Further research is to analyze convergence properties of such methods by using Theorem 3 given in this paper.

\section{Acknowledgement}

The authors would like to thank two referees for their valuable comments. 


\section{References}

[1] M. Al-Baali and R. Fletcher: Variational methods for non-linear least squares. Journal of the Operational Research Society, 36 (1985) 405-421.

[2] K. W. Brodlie, A. R. Gourlay and J. Greenstadt: Rank-one and rank-two corrections to positive definite matrices expressed in product form. Journal of the Institute of Mathematics and its Applications, 11 (1973) 73-82.

[3] C. G. Broyden, J. E. Dennis, Jr. and J. J. Moré: On the local and superlinear convergence of quasi-Newton methods. Journal of the Institute of Mathematics and its Applications, 12 (1973) 223-245.

[4] J. E. Dennis, Jr.: A brief survey of convergence results for quasi-Newton methods. SIAM-AMS Proceedings, 9 (1976) 185-199.

[5] J. E. Dennis, Jr. and J. J. Moré: A characterization of superlinear convergence and its application to quasi-Newton methods. Mathematics of Computation, 28 (1974) 549-560.

[6] J. E. Dennis, Jr. and R. B. Schnabel: A new derivation of symmetric positive definite secant updates. In O. L. Mangasarian, R. R. Meyer and S. M. Robinson (eds.): Nonlinear Programming 4 (Academic Press, New York, 1981) 167-199.

[7] J. E. Dennis, Jr. and R. B. Schnabel: Numerical Methods for Unconstrained Optimization and Nonlinear Equations (Prentice-Hall, Englewood Cliffs, NJ, 1983).

[8] J. E. Dennis, Jr., S. Sheng and P. A. Vu: A memoryless augmented Gauss-Newton method for nonlinear least-squares problems. Journal of Computational Mathematics, 6 (1988) 355-374.

[9] D. Gay: Representing symmetric rank 2 updates Working Paper, No.124 (Computer Research Center for Economics and Management Science, National Bureau of Economic Research, Inc., Cambridge, Massachusetts, 1976).

[10] D. Goldfarb: Factorized variable metric methods for unconstrained optimization. Mathematics of Computation, 30 (1976) 796-811.

[11] H. Y. Huang: Unified approach to quadratically convergent algorithms for function minimization. Journal of Optimization Theory and Applications, 5 (1970) 405-423.

[12] C. M. Ip: On least-change secant updates in factorized form. SIAM J. Numerical Analysis, 24 (1987) 1126-1132.

[13] A. Stachurski: Superlinear convergence of Broyden's bounded $\theta$-class of methods. Mathematical Programming, 20 (1981) 196-212.

[14] H. Yabe: A family of variable-metric methods with factorized expressions. TRU Mathematics, 17 (1981) 141-152.

[15] N. Yamaki and H. Yabe: On factorized variable-metric methods. TRU Mathematics, 17 (1981) 285-294.

[16] Y. Zhang and R. P. Tewarson: Least-change updates to Cholesky factors subject to the nonlinear quasi-Newton condition. IMA Journal of Numerical Analysis, 7 (1987) 509-521.

Hiroshi YABE

Department of Applied Mathematics,

Faculty of Science,

Science University of Tokyo,

1-3, Kagurazaka, Shinjuku-ku, Tokyo 162-8601, Japan

E-mail: yabe@am.kagu.sut.ac.jp 